\title{
An Introduction to Atom Probe Tomography
}

\author{
M. K. Miller* \\ *Oak Ridge National Laboratory, PO Box 2008, Oak Ridge, TN 37831-6136
}

Atom probe tomography (APT) is a microanalytical technique for the characterization of compositional variations in metals and semiconductors on the atomic scale [1]. APT has been applied to studies in many areas of material science including phase transformations, solute clustering, ordering, radiation damage, superalloys, intermetallics, bulk metallic glasses, thin films, multilayers and alloy design. APT is used primarily to study phase transformations, precipitation and segregation to microstructural features such as grain boundaries, interfaces and dislocations.

The main components and the principle of operation of a 3-dimensional atom probe (3DAP) are shown in Fig. 1. The needle-shaped specimen with a typical end radius of $\sim 50 \mathrm{~nm}$ is prepared by electropolishing or ion milling. Atoms are ionized and field evaporated from the apex of the cryogenically cooled $(20-70 \mathrm{~K})$ specimen by a high voltage pulse superimposed on a standing voltage. The elemental identities of these ions are determined from the mass-to-charge ratios, which are calculated from their flight times, $t$, in a time-of-flight mass spectrometer; $m / n=\operatorname{const} V t^{2} / d^{2}$. The $x$ and $y$ coordinates of each field evaporated atom are derived from the highly magnified $(\sim 5 \mathrm{x}$ $10^{6} \mathrm{X}$ ) impact position, $x_{1}$ and $y_{1}$, on the position-sensitive single atom detector. The $z$ coordinate is derived from the order in which the atom was detected. 3DAPs are also equipped with a microchannel plate and phosphor screen detector to view field ion images. Field ion microscopy is generally performed at the start of the experiment to ensure that the surface of the specimen has a well developed end form suitable for analysis and to assist in the selection of the initial area of analysis. The newest variant of 3DAP, the local electrode atom probe (LEAP), is equipped with a special XYZ stage to move the specimen relative to a closely positioned counter electrode. The lower operating voltage of the LEAP allows significantly higher atom collection rates and also permits local protrusions created by focused ion beam milling of flat surfaces to be individually selected and analyzed.

This tomographic technique produces 3D images of the internal structure of materials by the reconstruction of thousands of slices each containing a few atoms. The resulting data are the spatial coordinates and the elemental identities of the atoms in the sampled volume. Approximately $10^{5}$ to $10^{8}$ atoms are collected in each experiment. The mass resolution is sufficient to resolve the individual isotopes of all the elements. A variety of representations including atom maps, Fig. 2, isoconcentration surfaces, Fig. 3, 2D composition slices and concentration profiles may be used to visualize the $3 \mathrm{D}$ data. The atoms associated with particles down to the nanometer scale may be apportioned from the matrix atoms with the maximum separation method, as shown by the filled and open circles in Fig. 4. Their compositions may be estimated from the atoms contained in the shaded cells with the envelope method, Fig. 4. The atom-by-atom data may be statistically analyzed to estimate the solute clustering and ordering tendencies, solute partitioning into different phases, solute segregation to microstructural features such as grain boundaries, interfaces and dislocations, and some topological parameters [2].

[1] M.K. Miller, Atom Probe Tomography, Kluwer Academic/ Plenum, New York, 2000. 
[2] Research at the Oak Ridge National Laboratory SHaRE Collaborative Research Center was sponsored by the Division of Materials Sciences and Engineering, U.S. Department of Energy, under contract DE-AC05-00OR22725 with UT-Battelle, LLC.

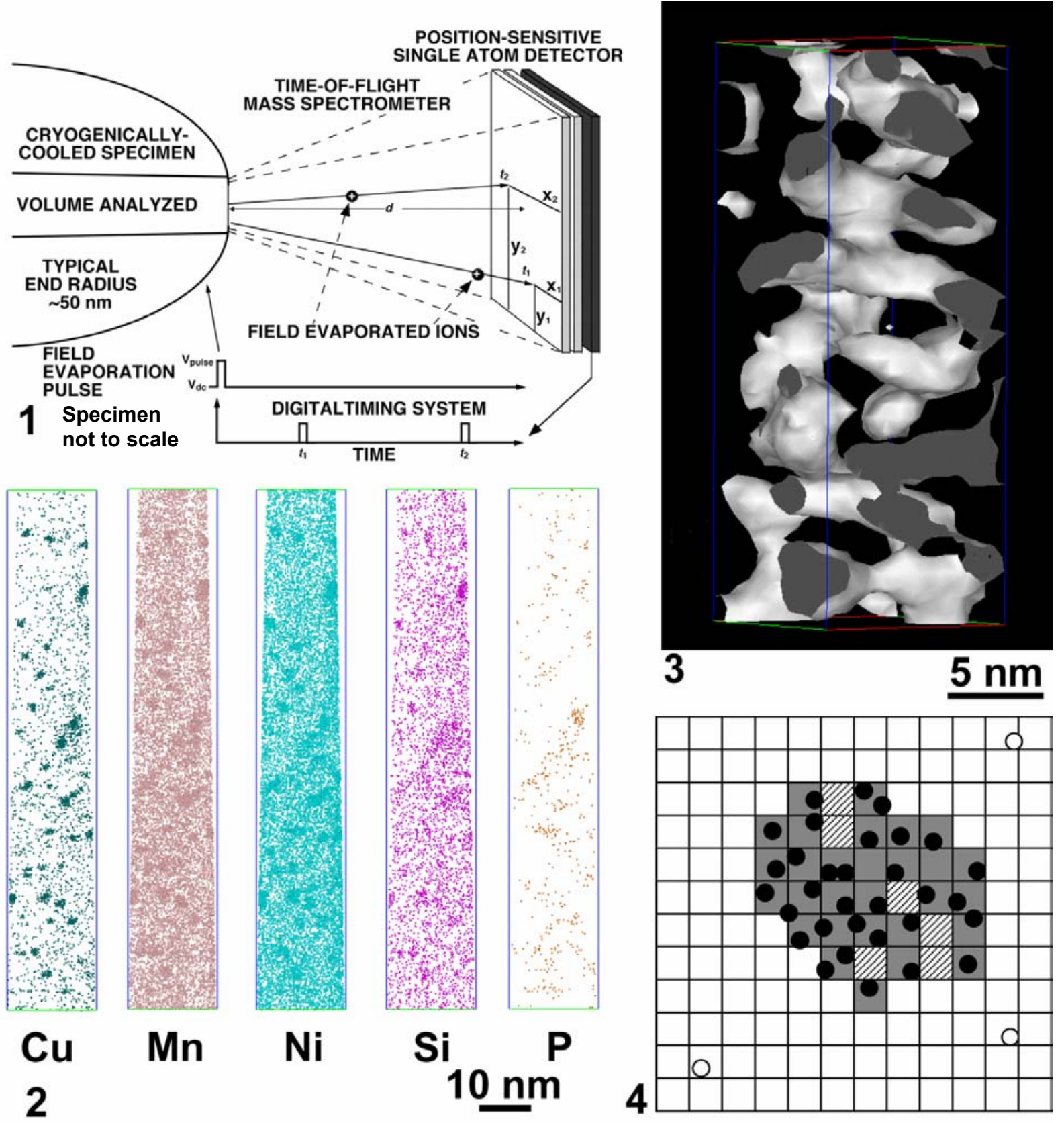

FIG. 1. A 3DAP features a time-of-flight mass spectrometer and a position-sensitive detector.

FIG. 2. Atom maps showing a high number density of 3-nm-diameter $\mathrm{Cu}-, \mathrm{Mn}-$, Ni-, Si- and Penriched precipitates in a neutron irradiated pressure vessel weld (KS01).

FIG. 3. Isoconcentration surface of the interconnected network structure of the Cr-enriched $\alpha$ phase in $\mathrm{Fe}-45 \% \mathrm{Cr}$ aged for $10,000 \mathrm{~h}$ at $470^{\circ} \mathrm{C}$.

FIG. 4. The maximum separation and envelope methods: the filled atoms defining the particle are selected based on their proximity to their neighbors; the particle composition is determined from all the atoms in the shaded region. 